\title{
Roman domination number on cardinal product of paths and cycles
}

\author{
Antoaneta Klobučar ${ }^{1, *}$ and Ivona Puljić ${ }^{2}$ \\ ${ }^{1}$ Faculty of Economics, Josip Juraj Strossmayer University of Osijek \\ $\operatorname{Trg} L j$. Gaja 7, 31000 Osijek, Croatia \\ E-mail: 〈aneta@efos.hr \\ ${ }^{2}$ Department of Mathematics, Josip Juraj Strossmayer University of Osijek \\ Trg Lj. Gaja 6, 31000 Osijek, Croatia \\ E-mail: 〈ivona.puljic@gmail.com〉
}

\begin{abstract}
In this paper, the authors have determined certain upper and lower bounds for Roman domination numbers on cardinal products for any two graphs and some exact values for the cardinal product of paths and cycles. Roman domination was named after the historical fact that Roman legions were distributed across regions during the reign of the Roman Emperor Constantine in the $4^{\text {th }}$ century A.D. Some areas had 1 or 2 legions, some had no legions, but every area had at least 1 neighboring area with 2 legions. Roman domination is used even today, not only in military situations, but also in protecting some locations against fire or crime.
\end{abstract}

Key words: Roman dominating function, Roman domination number $\gamma_{R}$, cardinal product of paths, cardinal product of paths and cycles

Received: October 4, 2014; accepted: February 15, 2015; available online: March 30, 2015

DOI:10.17535/crorr.2015.0006

\section{Historical background and basic definitions}

In the 4th century A.D., the Roman Empire was under the rule of Constantine the Great. During that time, the Empire suffered numerous barbaric attacks. Constantine saw that he had to arrange Roman legions in all the strategically important places for protection. Not only did this placement of Roman legions have to be successful in defending the Empire, it also had to be easy to maintain.

A location was considered secure if at least one Roman legion was stationed there. On the other hand, unsecure locations had no stationed legions, but had to be situated adjacent to at least one secure location. If an unsecure location was under attack, sending a legion from its secure neighbor would not be effective if it meant the neighboring location unsecure. Therefore, Constantine decreed that at least two legions had to be stationed at a location before one of them was sent to assist its neighbor under attack. In order to reduce costs of maintaining the army, Constantine had to use as few legions as possible, but still secure the whole Empire.

${ }^{*}$ Corresponding author. 
This historical background motivated Ian Stuart (1999) to suggest a new variant of the domination known as Roman domination. By representing locations in the Empire as graph vertices and roads in the Empire as graph edges, the problem of defending the Roman Empire transforms into a problem of protecting (or dominating) a graph.

$A$ dominating function on $G$ is any function $f: V \rightarrow\{0,1\}$ satisfying the condition that every vertex $u$ for which $f(u)=0$ is adjacent to at least one vertex $v$ for which $f(v)=1$. Such a function obviously induces the ordered partition $\left(V_{0}, V_{1}\right)$ of $V$ such that each vertex in $V_{0}$ is adjacent to at least one vertex in $V_{1}$. Therefore, the set $V_{1}$ is called a dominating set.

There is a bijection between the set of all functions $f: V \rightarrow\{0,1\}$ and the set of all ordered partitions $\left(V_{0}, V_{1}\right)$. Thus, the formulation $f=\left(V_{0}, V_{1}\right)$ is obtained. The weight of $f$ equals $w(f)=\sum_{v \in V} f(v)=0 \cdot\left|V_{0}\right|+1 \cdot\left|V_{1}\right|=\left|V_{1}\right|$. Obviously, the most interesting dominating functions are those of minimum weight.

For a graph $G=(V, E)$, a Roman dominating function (RDF) is a function $f: V \rightarrow\{0,1,2\}$ satisfying the condition that every vertex $u$ for which $f(u)=0$ is adjacent to at least one vertex $v$ for which $f(v)=2$. Since this function also induces the ordered partition of $V, V_{i}=\{v \in V: f(v)=i\}, i \in\{0,1,2\}$, the formulation $f=\left(V_{0}, V_{1}, V_{2}\right)$ becomes valid. The weight of an RDF equals $w(f)=\sum_{v \in V} f(v)=$ $0 \cdot\left|V_{0}\right|+1 \cdot\left|V_{1}\right|+2 \cdot\left|V_{2}\right|=\left|V_{1}\right|+2\left|V_{2}\right|$.

This definition implies that the same graph can be protected under several different RDFs, but the most important RDFs are those for which $w(f)$ achieves its minimum. This minimum weight is called the Roman domination number of $G$ and we denote it by $\gamma_{R}(G)$. An RDF which satisfies $w(f)=\gamma_{R}(G)$ is called $a$ $\gamma_{R}$-function. It is obvious that $\gamma_{R}(G) \leq\left|V_{1}\right|+2\left|V_{2}\right|$ for any $\operatorname{RDF} f=\left(V_{0}, V_{1}, V_{2}\right)$.

The main result describing a connection between domination and the Roman domination number of an arbitrary graph $G$ is

$$
\gamma(G) \leq \gamma_{R}(G) \leq 2 \gamma(G)
$$

Graphs for which $\gamma_{R}(G)=2 \gamma(G)$ are called Roman graphs. It is obvious that $\left|V_{1}\right|=0$ for every minimum weight RDF of any Roman graph.

Despite the fact that Roman domination has been studied for just a little over a decade, we already know the exact values of Roman domination numbers for many classes of graphs $([1],[2],[3])$. However, the complexity of some graphs allows the author(s) to establish only an upper bound.

For more on the domination parameters and the terminology, see [5].

For arbitrary graphs $G$ and $H$, the cardinal product of $G$ and $H$ is the graph $G \times H$ which satisfies the following

- Its vertex set is $V(G \times H)=V(G) \times V(H)$;

- Two vertices $(g, h),\left(g^{\prime}, h^{\prime}\right) \in V(G \times H)$ are adjacent if and only if $g$ is adjacent to $g^{\prime}$ in $G$ and $h$ is adjacent to $h^{\prime}$ in $H$.

The cardinal product of two paths $P_{m} \times P_{n}$ has two connected components. If the vertices of $P_{m}$ and $P_{n}$ are denoted by $\{1,2,3, \ldots, m\}$ and $\{1,2,3, \ldots, n\}$, respectively, then the component of $P_{m} \times P_{n}$ containing the vertex $(1,1)$ will be denoted 
by $K_{1}$, and the other component by $K_{2}$. If at least one of the parameters $m$ or $n$ is even, components $K_{1}$ and $K_{2}$ are isomorphic. Otherwise, the component $K_{1}$ has one vertex more than the component $K_{2}$.

In [1] we proved bounds for Roman domination numbers of the cardinal product of two paths and two cycles. Now, some bounds for cardinal product of any two graphs will be provided, and will take the exact values of Roman domination numbers for products of some graphs..

\section{Bounds for Roman domination numbers on the cardinal product}

The degree of a vertex $x$ on graph $G$ is the number of vertices on $G$ which are adjacent to $x$. Using $\Delta(G)(\delta(G))$ we denote the maximum (minimum) degree of vertices on $G$.

Lemma 1. In [2], it has been proven that for any graph $G$ of order $n$

$$
\begin{gathered}
\gamma_{R}(G) \geq \frac{2 n}{\Delta+1} \\
\gamma_{R}(G) \leq n \frac{2+\ln ((1+\delta(G)) / 2)}{\delta(G)+1}
\end{gathered}
$$

Theorem 1. For any two graphs $G$ and $H$ of order $m$ and $n$

$$
\begin{gathered}
\gamma_{R}(G \times H) \geq \frac{2 m n}{\Delta(G) \Delta(H)+1} \\
\gamma_{R}(G \times H) \leq m n \frac{2+\ln ((1+\delta(G) \delta(H)) / 2)}{\delta(G) \delta(H))+1}
\end{gathered}
$$

Proof. According to the definition of the cardinal product, vertex $(i, j) \in$ $V(G \times H), i \in V(G), j \in V(H)$, is adjacent to $z w$ vertices in graph $G \times H$, where $z$ is the number of the vertices which are adjacent to $i$ in graph $G$, and $w$ is the number of the vertices which are adjacent to $j$ in graph $H$, which implies that $\Delta(G \times H)=\Delta(G) \times \Delta(H)$. (The same is true for minimal degree.) But, since graph $G \times H$ is of order $m n$, the statement is true based on the previous Lemma.

Here, the exact values of Roman domination numbers for the products of certain graphs will be taken.

\section{Specific values of Roman domination numbers}

Observation 1. Let $C_{n}$ and $P_{n}$ denote the cycle and path with $n$ vertices, respectively. Then

$$
\gamma_{R}\left(C_{n}\right)=\gamma_{R}\left(P_{n}\right)=\left\lceil\frac{2 n}{3}\right\rceil .
$$


Following the investigations into the Cartesian product, cardinal products where one of the factors is a path are considered.

Theorem 2. For any tree $T$ and any graph $G$ without cycles of odd length we have

$$
\gamma_{R}\left(P_{2} \times T\right)=2 \gamma_{R}(T)=\gamma_{R}\left(P_{2}\right) \gamma_{R}(T)
$$

and

$$
\gamma_{R}\left(P_{2} \times G\right)=2 \gamma_{R}(G)=\gamma_{R}\left(P_{2}\right) \gamma_{R}(G) .
$$

Proof. Obvious, since $P_{2} \times T$ and $P_{2} \times G$ consist of two disjoint copies of $T$ and $G$, respectively

Theorem 3. For the path $P_{2}$ and any odd cycle $C_{2 n+1}, n \geq 1$,

$$
\gamma_{R}\left(P_{2} \times C_{2 n+1}\right)=\left\lceil\frac{2(4 n+2)}{3}\right\rceil=2\left\lceil\frac{4 n+2}{3}\right\rceil=\gamma_{R}\left(P_{2}\right) \gamma_{R}\left(C_{2 n+1}\right)
$$

Proof. Note that the cardinal product of $P_{2}$ and $C_{2 n+1}$ is isomorphic to $C_{4 n+2}$. Then Observation 1 implies that

$$
\gamma_{R}\left(C_{4 n+2}\right)=\left\lceil\frac{2(4 n+2)}{3}\right\rceil=2\left\lceil\frac{2 n+1}{3}\right\rceil=\gamma_{R}\left(P_{2}\right) \gamma_{R}\left(C_{2 n+1}\right)
$$

Definition 1. For a fixed $m, 1 \leq m \leq n$, the set $\left(P_{k}\right)_{m}=\{(i, m) \mid i=1, \ldots, k\}$ is called a column of $P_{k} \times P_{n}$. For a fixed $l, 1 \leq l \leq k$, The set $\left(P_{n}\right)_{l}=\{(l, j) \mid j=$ $1, \ldots, n\}$ is called a row of $P_{k} \times P_{n}$.

Theorem 4. Let $n \geq 2$. Then

$$
\gamma_{R}\left(P_{3} \times P_{n}\right)=\left\{\begin{aligned}
& 6\left(\frac{n}{4}\right) n \equiv 0(\bmod 4), \\
& 6\left\lfloor\frac{n}{4}\right\rfloor+2 n \equiv 1(\bmod 4), \\
& 6\left\lfloor\frac{n}{4}\right\rfloor+4 n \equiv 2(\bmod 4), \\
& 6\left\lfloor\frac{n}{4}\right\rfloor+5 n \equiv 3(\bmod 4)
\end{aligned}\right.
$$

Proof. First, it is necessary to find the upper bound for $\gamma_{R}\left(P_{3} \times P_{n}\right)$ by constructing some Roman dominating sets. Later, the minimality will be proven.

Case 1. $n \equiv 0(\bmod 4)$

Since, for this case, $P_{3} \times P_{n}$ consists of two isomorphic components, we consider only $K_{1}$. Set $S_{1}=\left\{\left((2,4 j-2) \in V_{2} ; j=1, \ldots, \frac{n}{4}\right) \cup\left((2,4 j) \in V_{1} ; j=1, \ldots, \frac{n}{4}\right)\right\}$ is a Roman dominating set and hence for $n \equiv 0(\bmod 4)$, $\gamma_{R}\left(P_{3} \times P_{n}\right) \leq 2\left|S_{1}\right|=2\left(3 \frac{n}{4}\right)=6 \frac{n}{4}$.

Case 2. $n \equiv 1(\bmod 4)$

On $K_{1}$, the set $S_{1}=\left\{\left((2,4 j-2) \cup(2, n-1) \in V_{2} ; j=1, \ldots,\left\lfloor\frac{n}{4}\right\rfloor\right) \cup((2,4 j) \in\right.$ $\left.\left.V_{1} ; j=1, \ldots,\left\lfloor\frac{n}{4}\right\rfloor-1\right)\right\}$ is deemed to be a Roman dominating set, and on $K_{2}$ the set $S_{2}=\left\{\left((2,4 j-1) \in V_{2} ; j=1, \ldots,\left\lfloor\frac{n}{4}\right\rfloor\right) \cup\left((2,4 j-3) \cup(2, n) \in V_{1} ; j=1, \ldots,\left\lfloor\frac{n}{4}\right\rfloor\right)\right\}$ is a Roman dominating set.

Hence for $n \equiv 1(\bmod 4), \gamma_{R}\left(P_{3} \times P_{n}\right) \leq\left|S_{1}\right| \cup\left|S_{2}\right|=2\left(3\left\lfloor\frac{n}{4}\right\rfloor+1\right)=6\left\lfloor\frac{n}{4}\right\rfloor+2$. 
Case 3. $n \equiv 2(\bmod 4)$

Since, for this case, $P_{3} \times P_{n}$ consists of two isomorphic components, only $K_{1}$ is considered. Set $S_{1}=\left\{\left((2,4 j-2) \cup(2, n) \in V_{2} ; j=1, \ldots,\left\lfloor\frac{n}{4}\right\rfloor\right) \cup\left((2,4 j) \in V_{1} ; j=1, \ldots,\left\lfloor\frac{n}{4}\right\rfloor\right)\right\}$ is a Roman dominating set and hence for $n \equiv 2(\bmod 4)$, $\gamma_{R}\left(P_{3} \times P_{n}\right) \leq 2\left|S_{1}\right|=2\left(3\left\lfloor\frac{n}{4}\right\rfloor+2\right)=6\left\lfloor\frac{n}{4}\right\rfloor+4$.

Case 4. $n \equiv 3(\bmod 4)$

On $K_{1}$, the set $S_{1}=\left\{\left((2,4 j-2) \cup(2, n-1) \in V_{2} ; j=1, \ldots,\left\lfloor\frac{n}{4}\right\rfloor\right) \cup((2,4 j) \in\right.$ $\left.\left.V_{1} ; j=1, \ldots,\left\lfloor\frac{n}{4}\right\rfloor\right)\right\}$ is considered a Roman dominating set, and on $K_{2}$ the set $S_{2}=\left\{\left((2,4 j-1) \cup(2, n) \in V_{2} ; j=1, \ldots,\left\lfloor\frac{n}{4}\right\rfloor\right) \cup\left((2,4 j-3) \in V_{1} ; j=1, \ldots,\left\lfloor\frac{n}{4}\right\rfloor+1\right)\right\}$ is a Roman dominating set, hence for $n \equiv 3(\bmod 4)$,

$\gamma_{R}\left(P_{3} \times P_{n}\right) \leq\left|S_{1}\right| \cup\left|S_{2}\right|=\left(3\left\lfloor\frac{n}{4}\right\rfloor+2\right)+\left(3\left\lfloor\frac{n}{4}\right\rfloor+3\right)=6\left\lfloor\frac{n}{4}\right\rfloor+5$.

We now prove that

$$
\gamma_{R}\left(P_{3} \times P_{n}\right) \geq\left\{\begin{aligned}
& 6\left(\frac{n}{4}\right) n \equiv 0(\bmod 4) \\
& 6\left\lfloor\frac{n}{4}\right\rfloor+2 n \equiv 1(\bmod 4), \\
& 6\left\lfloor\frac{n}{4}\right\rfloor+4 n \equiv 2(\bmod 4) \\
& 6\left\lfloor\frac{n}{4}\right\rfloor+5 n \equiv 3(\bmod 4)
\end{aligned}\right.
$$

Proof of the minimality:

Case 1. Let $n$ is even.

We will consider the component $K_{1}$.

Lemma 2. There is a minimum Roman dominating set $D$, such that $D$ only contains vertices of the row (2,i), $i \in\{2,4, \ldots, n\}$.

Proof (of the Lemma). Let $D$ be a minimal Roman dominating set that does not satisfy the assertion. Without loss of generality, $D$ is assumed to contain a vertex of the row $\left(P_{n}\right)_{1}$. Let $(1, j)$ be this vertex for some fixed $j \in\{3, \ldots, n-1\}$.

If $(1, j) \in V_{1}$ and $(3, j) \notin D$ then $(2, j-1)$ or $(2, j+1) \in V_{2}$. Then the set $D^{\prime}=D \backslash(1, j)$ also Roman dominates $K_{1}$ and $\left|D^{\prime}\right| \leq|D|$. So, $D$ is not minimal.

If $(1, j) \in V_{1}$ and $(3, j) \in V_{1}$ then $(2, j-1)$ or $(2, j+1) \in D$.

a) Let $(2, j+1) \in V_{2}$ Then the set $D^{\prime}=\{D \backslash((1, j),(3, j))\}$ also Roman dominates $K_{1}$. So, $D$ is not minimal.

b) If $(2, j+1) \in V_{1}$, we can also take the same set $D^{\prime}$ as in a), and $D$ is not minimal.

If $(1, j) \in V_{1}$ and $(3, j) \in V_{2}$, Then the set $D^{\prime}=(D \backslash\{(1, j),(3, j)\}) \cup\{(2, j-1) \in$ $\left.V_{1},(2, j+1) \in V_{2}\right\}$ also Roman dominates $K_{1}$.

If $(1, j) \in V_{2}$ and $(3, j) \notin D$ then $(2, j-1)$ or $(2, j+1) \in V_{2}$. Let $(2, j+1) \in V_{2}$. Then the set $D^{\prime}=D \backslash(1, j) \cup\left((2, j-1) \in V_{2}\right)$ also Roman dominates $K_{1}$.

If $(1, j) \in V_{2}$ and $(3, j) \in V_{1}$, then the set $D^{\prime}=\{(D \backslash(1, j),(3, j)) \cup((2, j+1) \in$ $\left.\left.V_{2},(2, j-1) \in V_{1}\right)\right\}$ also Roman dominates $K_{1}$. 
If $(1, j) \in V_{2}$ and $(3, j) \in V_{2}$, Then the set $D^{\prime}=(D \backslash\{(1, j),(3, j)\}) \cup\{((2, j-$ 1), $\left.\left.(2, j+1) \in V_{2}\right)\right\}$ also Roman dominates $K_{1}$.

For $(1,1) \in D$ is similarly.

Case 2. Let $n$ be odd.

For both components, the assertion of Lemma 1 can be shown analogously.

From Lemma 1 and condition that each vertex from $V_{o}$ must be adjacent to at least one vertex from $V_{2}$, it follows that, to R-dominate vertices from the first and last row (on $\left.K_{1}\right)$, at least vertices $\left\{\left((2,4 j-2) ; j=1, \ldots,\left\lfloor\frac{n}{4}\right\rfloor\right)\right\}$ must be in $V_{2}$, and then the remaining vertices on row $(2, \mathrm{i})$ must be in $V_{1}$. (For $K_{2}$ this is similar.) And this is exactly the structure from the set $S=S_{1} \cup S_{2}$.

Theorem 5. Let $n \geq 2$. Then

$$
\gamma_{R}\left(P_{4} \times P_{n}\right)=\left\{\begin{array}{cl}
2 n+2 & n=5 \\
2 n & \text { otherwise }
\end{array}\right.
$$

\section{Proof.}

Since $P_{4} \times P_{n}$ consists of two isomorphic components, only $K_{1}$ is considered.

Case 1. $n \equiv 0(\bmod 6)$

Set $S_{1}=\left\{(2,6 j+2),(3,6 j+6): j=0,1, \ldots,\left\lfloor\frac{n}{6}\right\rfloor-1\right) \in V_{2} ; \cup(1,6 j+6),(4,6 j+2)$ : $\left.\left.j=0,1, \ldots,\left\lfloor\frac{n}{6}\right\rfloor-1\right) \in V_{1}\right\}$ is Roman dominating set and hence for $n \equiv 0(\bmod 6)$, $\gamma_{R}\left(P_{4} \times P_{n}\right) \leq 2\left|S_{1}\right|=2(n)$.

Case 2. $n \equiv 1(\bmod 6)$

The set $S_{1}^{\prime}=\left(S_{1} \backslash\left((1, n-2) \in V_{1}\right) \cup\left((2, n-1) \in V_{2}\right)\right)$ is considered. This set is a Roman dominating set and hence for $n \equiv 1(\bmod 6)$,

$\gamma_{R}\left(P_{4} \times P_{n}\right) \leq 2\left|S_{1}^{\prime}\right|=2(n)$.

Case 3. $n \equiv 2(\bmod 6)$

The set $S_{1}^{\prime}=\left(S_{1} \backslash\left(\{(4, n-6),(1, n-3)\} \in V_{1},(3, n-3) \in V_{2}\right) \cup(\{(3, n-5),(2, n-\right.$ $\left.\left.2),(3, n-1) \in V_{2}\right)\right)$ is considered. This set is a Roman dominating set and hence for $n \equiv 2(\bmod 6)$,

$\gamma_{R}\left(P_{4} \times P_{n}\right) \leq 2\left|S_{1}^{\prime}\right|=2(n)$.

Case 4. $n \equiv 3(\bmod 6)$

The set $S_{1}^{\prime}=\left(S_{1} \cup\left((2, n) \in V_{2},(4, n) \in V_{1}\right)\right.$ is considered. This set is a Roman dominating set and hence for $n \equiv 3(\bmod 6)$, $\gamma_{R}\left(P_{4} \times P_{n}\right) \leq 2\left|S_{1}^{\prime}\right|=2(n)$.

Case 5. $n \equiv 4(\bmod 6)$

The set $S_{1}^{\prime}=\left(S_{1} \cup\left((2, n-2),(3, n-1) \in V_{2}\right)\right)$ is considered. This set is a Roman dominating set and hence for $n \equiv 4(\bmod 6)$, $\gamma_{R}\left(P_{4} \times P_{n}\right) \leq 2\left|S_{1}^{\prime}\right|=2(n)$. 
Case 6. $n \equiv 5(\bmod 6)$

The set $S_{1}^{\prime}=\left(S_{1} \backslash\left((4, n-6) \in V_{1}\right) \cup\left((3, n-7),(3, n-2),(2, n-1) \in V_{2}\right)\right.$ is considered. This set is a Roman dominating set and hence for $n \equiv 5(\bmod 6)$, $\gamma_{R}\left(P_{4} \times P_{n}\right) \leq 2\left|S_{1}^{\prime}\right|=2(n)$.

Proof of minimality:

Let $n \equiv 0(\bmod 6)($ or $n \equiv 3(\bmod 6))$. If we partition the graph $P_{4} \times P_{n}$ into $4 \times 3$ blocks, it follows that in $S_{1}$, on each such block there exists 1 vertex from $V_{2}$ (weight 2 ) which Roman dominates 5 vertices, and 1 vertex from $V_{1}$ (weight 1 ) which Roman dominates only itself, and it lies in the first or the last row. In [1] it has been proved that the optimal Roman dominating set has exactly such structure. For other cases of $n$ each new column increases the weight of the graph by 1 . A smaller increase is not possible.

For $P_{5} \times P_{n}$ and $P_{6} \times P_{n}$ we have the following bounds:

$$
\begin{aligned}
\gamma_{R}\left(P_{5} \times P_{n}\right) \leq\left\{\begin{array}{rl}
8\left(\frac{n}{3}\right) & n \equiv 0(\bmod 3) \\
8\left\lfloor\frac{n}{3}\right\rfloor+4 n & \equiv 1(\bmod 3), \\
8\left\lceil\frac{n}{3}\right\rceil & n \equiv 2(\bmod 4) \\
10\left(\frac{n}{3}\right) & n \equiv 0(\bmod 3), \\
10\left\lfloor\frac{n}{3}\right\rfloor+2 n & \equiv 1(\bmod 3), \\
10\left\lfloor\frac{n}{3}\right\rfloor+4 & n \equiv 2(\bmod 3)
\end{array}\right. \\
\gamma_{R}\left(P_{6} \times P_{n}\right)
\end{aligned}
$$

We also have the conjecture that equality holds in both bounds, but still remains to be proven.

\section{Conclusion}

This paper provides bounds of Roman domination numbers for the product of any two graphs. Furthermore, exact values (not bounds) are given for the product of $P_{2}$ with any graph, and for $P_{3} \times P_{n}$ and $P_{4} \times P_{n}$. Upper bounds for the Roman domination number of $P_{5} \times P_{n}$ and $P_{6} \times P_{n}$ are also given, and conjecture is that they are exact values. This paper is a continuation of the paper in [1]. Bounds have been given only for Roman domination numbers of the cardinal product of two paths and two cycles.

Even today, Roman domination can be applied in the real life, and not only in a military sense. For instance, in unsecure parts of a town, where police are often called to intervene, there should be at least two teams of police. Meaning that when, one team goes out, the other team can react to another call. This service arrangement already exists in emergency medical stations. Such stations have several teams ready to intervene. The Roman domination can even be generalized by postulating that 3 or more teams dominate an area.

\section{References}

[1] Klobučar A. and Puljić, I. (2014). Some results for Roman domination number on Cardinal product of paths and cycles. Kragujevac Journal of Mathematics, 38(1), 
83-94.

[2] Cockayne, E.J., Dreyer Jr., P.A., Hedetniemi, S.M. and Hedetniemi, S.T. (2004). Roman domination in graphs. Discrete Math. 278, 11-22.

[3] Favaron, O., Karami, H., Khoeilar, R. and Sheikholeslami, S.M. (2009). On the Roman domination number of a graph. Discrete Math. 309, 3447-3451. doi:10.1016/j.disc.2008.09.043.

[4] Cockayne, E.J., Grobler, P.J.P., Gründlingh, W.R., Munganga, J. and van Vuuren, J.H. (2005). Protection of a graph. Util. Math. 67, 19-32.

[5] Haynes, T.W., Hedetniemi, S.T. and Slater, P.J. (1999). Fundamental of Domination in Graphs. Marcel Deker, Inc. New York

[6] Klobučar, A. (2005). On the k-dominating number of Cartesian products of two paths. Math. Slovaca 55, 141-154.

[7] Klobučar, A. (2001). K-dominating sets of $P_{2 k+2} \times P_{n}$ and $P_{m} \times P_{n}$. Ars Combin. 58, 279-288.

[8] Klobučar, A. (1999). Domination numbers of cardinal products. Math. Slovaca 49, 387-402.

[9] Klobučar, A. and Seifter, N. (2000). K-dominating sets of cardinal products of paths. Ars Combin. 55, 33-41.

[10] Pavlič, P. and Žerovnik, J. (2012). Roman domination number of the Cartesian products of paths and cycles. The Electronic Journal of Combinatorics, 19, Paper P19. 